TRABAJOS ORIGINALES

Rev Obstet Ginecol Venez. 2020; 80 (4): 303-311

https://doi.org/10.51288/00800407

\title{
Ecografía pélvica: correlación de la evolución de útero y ovarios con estadios de Tanner de mama y edad*
}

\author{
Drs. Priscila Rodríguez, ${ }^{1}$ María Mercedes Pérez, ${ }^{1}$ Adelvi Nieto, ${ }^{1}$ Alfredo Caraballo, ${ }^{1}$ \\ Melissa Osuna. ${ }^{1}$
}

\begin{abstract}
RESUMEN
Objetivo: Correlacionar la evolución ecográfica de útero y ovarios, según los estadios de Tanner de mama y la edad cronológica en pacientes que acudieron a la consulta de ginecología infantil y juvenil del hospital de niños "Dr. José Manuel de los Ríos" entre marzo y octubre de 2016.

Métodos: Se evaluaron 113 pacientes con edades entre 7,7 y 15,5 años, excluyendo aquellas que habían presentado la menarquia, portadoras de malformación uroginecológica, con antecedente de cirugía uterina u ovárica o diagnóstico de alguna endocrinopatía. Se clasificaron según estadios de Tanner de mama y se les realizó ecografía pélvica transabdominal, se midió y describió útero y ovarios. Se calculó la media, desviación estándar y mediana según el tipo de variable, se aplicó ANOVA, prueba no paramétrica H de Kruskal-Wallis y chi-cuadrado de Pearson, se consideró un valor estadísticamente significativo si p < 0,05.

Resultados: La longitud uterina varió desde $33 \mathrm{~mm}$ en pacientes con Tanner I hasta $52 \mathrm{~mm}$ en aquellas con Tanner IV, la relación cuerpo/cuello fue de 0,9 en pacientes con estadio I; 1,12 en estadio II; 1,42 estadio III y 1,30 estadio IV. Se encontró relación estadísticamente significativa entre los volúmenes ováricos tanto con los grupos etarios como con los estadios de Tanner. En cuanto al patrón ovárico, el más frecuente observado fue el microfolicular.

Conclusiones: El útero y los ovarios presentan crecimiento continuo en relación a la edad y al estadio de Tanner de mama, factores con los cuales se demostró relación estadísticamente significativa.
\end{abstract}

Palabras clave: Ecografia pélvica, Útero, Ovarios, Estadios de Tanner de mama.

\section{SUMMARY}

Objective: To correlate the ultrasound evolution of the uterus and ovaries, according to Tanner's stages of breast and chronological age in patients attending the children's and juvenile gynecology clinic of the children's hospital "Dr. José Manuel de los Ríos "between March and October 2016.

Methods: 113 patients aged between 7.7 and 15.5 years were evaluated. From them were excluded those ones who had presented menarche, urogynecologic malformation, endocrinopathy or a history of uterine or ovarian surgery. They were classified according to Tanner stages of breast. Transabdominal pelvic ultrasound was performed, additionally uterus and ovaries were measured and described. We calculated the mean standard deviation and median according to the type of variable, we applied an ANOVA non-parametric test of KruskalWallis and chi-square of Pearson, it can be considered a statistically significant value if $p<0.05$.

Results: Uterine length ranged from $33 \mathrm{~mm}$ in patients with Tanner I up to $52 \mathrm{~mm}$ in those with Tanner IV. Body/cervix ratio was 0.9 in patients with stage I, 1.12 with stage II, 1.42 with stage III and 1.30 with stage IV. A statistically significant relationship was found between ovarian volumes with both age groups and Tanner stages. As for the ovarian pattern, the most frequent one was the microfollicular.

Conclusions: The uterus and ovaries show continuous growth in relation to age and the Tanner stage of breast, those factors with which a statistically significant relationship was demonstrated.

Keywords: Pelvic ultrasound, uterus, ovaries, Tanner stages of breast.

\footnotetext{
${ }^{1}$ Servicio de Ginecología Infantil y Juvenil Hospital de Niños “Dr. José Manuel de los Ríos"

* Trabajo Especial de Grado presentado por Priscila Rodríguez para optar al título de especialista en Ginecología Infantil y Juvenil. Trabajo presentado ante la Sociedad de Obstetricia y Ginecología de Venezuela para optar a miembro titular.
}

\section{INTRODUCCIÓN}

La ecografía es un método no invasivo e indoloro, no ionizante y de bajo costo, necesario para la evaluación

Vol. 80, № 4, diciembre 2020. 
en casi todos los cuadros clínicos que se presentan en el marco de la ginecología pediátrica y de la adolescencia (1). Algunas indicaciones para el empleo de ecografía pélvica durante esta etapa de la vida incluyen: descartar origen ginecológico en dolor abdominal, como complemento de la exploración ginecológica en estudios de trastornos funcionales y del desarrollo, como amenorrea primaria, pubertad precoz o tardía, y monitoreo en el tratamiento de las mismas (1). En muchas de las niñas es la única modalidad de imagen necesaria, pudiendo cambiar el diagnóstico clínico inicial y las decisiones terapéuticas (2).

La pubertad tiene una gran variabilidad individual, tanto en la edad de inicio como en su evolución, y depende fundamentalmente de la interacción de factores genéticos con el medio ambiente nutricional, psicosocial y climático. En la niña, involucra una secuencia de cambios físicos a lo largo de un periodo aproximado de 3 a 4 años, originados por un aumento gradual de los esteroides sexuales $(3,4)$.

En general, el primer signo puberal es la aceleración del crecimiento, seguida del brote de las mamas (telarquia) y aunque la secuencia puede invertirse, la adrenarquia suele aparecer después, siendo la menarquia un acontecimiento tardío, que ocurre una vez sobrepasado el pico del crecimiento. La telarquia es dependiente de la influencia estrogénica proveniente de los ovarios (5) y los cambios por los que atraviesan las mamas fueron descritos por Tanner: estadio 1: aspecto infantil; estadio 2: elevación mamaria y papilar en forma de un pequeño montículo; areola aumentada de diámetro y luce pigmentada; estadio 3: agrandamiento adicional con elevación de mamas y areolas en un mismo plano; estadio 4: continúa el crecimiento, la areola y pezón sobresalen del resto de la pirámide mamaria como una segunda elevación; estadio 5: mama de configuración adulta, no hay separación de la areola del resto de la pirámide mamaria, estando en un mismo plano; solo se proyecta el pezón (6).
Otros cambios en este periodo puberal ocurren en los genitales internos, ya que con la exposición a los estrógenos se produce el desarrollo del botón mamario ya mencionado, pero también la estrogenización de la mucosa vaginal y el crecimiento en volumen y longitud del útero (6), el cuerpo del útero empieza a crecer más rápido que el cuello y para el momento de la menarquia el diámetro mayor del cuerpo alcanza 1 y 1,5 veces el del cuello (7).

Durante la evaluación ecográfica en la pubertad, se pueden identificar los siguientes criterios de impregnación estrogénica del útero: 1) Aumento de la longitud del útero que supera los $3,5 \mathrm{~cm}$. 2) Engrosamiento del cuerpo uterino y relación cuerpo/ cuello mayor de uno. 3) Visibilidad de la línea endometrial (8 - 10). En cuanto a los ovarios, su volumen se mantiene sin cambios durante los primeros 2 años de vida y solo muestra un pequeño aumento hasta la pubertad, cuando hay un incremento constante y marcado del volumen (2). No resulta sencillo definir con precisión los criterios de maduración ovárica, ya que se han detectado folículos a partir de los 3 años de edad que no tienen relación con el inicio puberal. Algunos autores describen un aspecto microfolicular a partir de los 6 meses de edad (8), sin embargo se puede describir en la niña premenárquica, entre los 2 y 12 años de edad, que el ovario tiene aspecto ecográfico heterogéneo, debido a la presencia de folículos de tamaño variable (2). Salardi y cols. (11), en 1985, describieron las características ecográficas ováricas de la siguiente manera: Homogénea: estructuras ecolúcidas de tamaño $\leq 2 \mathrm{~mm}$. No homogénea: máximo 3 estructuras ecolúcidas que midan entre 3 y $8 \mathrm{~mm}$. Microfolicular: 4 o más estructuras ecolúcidas $<8 \mathrm{~mm}$. Folicular: estructuras ecolúcidas entre 9 y 25 mm. Quística: estructura ecolúcida $>25 \mathrm{~mm}$.

En la literatura internacional se encuentran distintos autores que han evaluado ecográficamente las dimensiones y ecogenicidad uterina y ováricas, 
comparándolas con la edad y estatus puberal, así como con edad ósea y otros aspectos como peso y talla, entre estos Herter y cols. (12), en Brasil, realizaron un estudio prospectivo entre agosto de 1998 y abril de 1999, donde incluyeron 139 pacientes de edades comprendidas entre 1 y 13 años de edad y evaluaron la correlación entre el volumen y la morfología de los ovarios, el volumen, el área y la longitud del útero, observados a la ecografía, con la edad cronológica, edad ósea y estadio puberal de mama y encontraron que sí existe correlación entre los factores antes mencionados; demostraron la influencia gonadotropa en la morfología ovárica y que las modificaciones en volumen y longitud uterina difieren en pacientes de iguales edades, con y sin telarquia. Más adelante, entre febrero de 2007 y febrero de 2009, RazzaghyAzar y cols. (13) evaluaron a 240 pacientes para establecer valores normales de referencia de medidas uterinas y ováricas en pacientes sanas de entre 6 y 13 años de edad, según el estadio puberal; encontraron un aumento gradual con la edad, en todas las medidas uterinas y ováricas. Además observaron una relación significativa entre los parámetros uterinos y ováricos con la edad, peso, estatura y estado puberal. En la literatura nacional, Caraballo y cols. (14), en 2002, estudiaron 685 pacientes de edades entre 2 semanas y 19 años, de las cuales se tomaron 600 para determinar medidas ultrasonográficas de útero y ovarios, estableciendo tablas de referencia en prepúberes y adolescentes.

Debido a lo antes mencionado, se pueden encontrar características ecográficas diferentes entre individuos de la misma edad que han tenido influencia hormonal distinta, por lo que diferenciar los casos que ameriten intervención de aquellos que no ameritan, implica un reto para el médico tratante. De allí la importancia de contar con parámetros de referencia de normalidad para poder hacer esta distinción. El presente estudio se hizo con el objetivo de correlacionar la evolución ecográfica de útero y ovarios, de acuerdo a los estadios de Tanner de mama y la edad cronológica.

\section{MÉTODOS}

Se realizó un estudio longitudinal, prospectivo, observacional, descriptivo y de correlación. La población estuvo conformada por pacientes femeninos con edades comprendidas entre 7,7 y 15,5 años, con desarrollo mamario según clasificación de Tanner en estadios I, II, III y IV, que no habían presentado menarquia y asistieron a la consulta del Servicio de Ginecología Infantily Juvenil del Hospital de Niños "Dr. José Manuel de los Ríos”, en el periodo comprendido entre el 1 de marzo de 2016 y 31 de agosto del mismo año. Se excluyeron aquellas pacientes portadoras de alguna malformación uroginecológica, con antecedente de cirugía uterina u ovárica o diagnóstico de alguna endocrinopatía o condición y tratamiento médico que pudiera afectar el inicio y desarrollo puberal. Se les informó acerca de las características y alcance del estudio, entregándoseles también el asentimiento y consentimiento informado por escrito para su lectura, comprensión y libre aprobación; posteriormente se examinaron para determinar estadio del desarrollo mamario según la clasificación de Tanner. Una vez incluidas en el estudio, las pacientes quienes tenían la vejiga llena (por ingesta de líquidos), se acostaron en posición decúbito dorsal y se procedió a realizar la evaluación ecográfica (la cual se realizó para fines de este estudio), con el uso de un equipo de ultrasonido en tiempo real, marca Aloka Co, LTD, modelo IPC1530, Tokio, Japón, transductor multifrecuencial de 3,5 $\mathrm{MHz}$ convexo. Se utilizó un agente de contacto en forma de gel acuoso entre el transductor y la piel del abdomen (previo descarte de alergia colocando 1 cc en la piel para observar reacción en los próximos 5 minutos). Al identificar el útero, con el transductor de forma que el eje mayor del mismo coincidiera con el eje mayor de la paciente, se realizaron las medidas longitudinales (diferenciando el cuerpo del cuello uterino) y anteroposteriores, evaluando en este mismo corte la línea endometrial. A continuación, se rotó el transductor $90^{\circ}$ para la medida transversal del útero, 
seguidamente se describió el ecopatrón miometrial y los contornos del útero. Con estas medidas se calculó el volumen uterino empleando la fórmula del elipsoide (vol. en $\mathrm{cm}^{3}=$ diámetro longitudinal en $\mathrm{cm} \mathrm{x}$ diámetro anteroposterior en $\mathrm{cm} \times$ diámetro transverso en $\mathrm{cm}$ $\mathrm{x} 0,523)$. Se ubicaron los ovarios y se realizaron las medidas de sus diámetros longitudinal, anteroposterior y transversal, con dichas medidas se empleó la misma fórmula de cálculo de volumen del elipsoide para determinar el volumen ovárico, además se describió el ecopatrón ovárico. Una vez obtenidos estos datos fueron asentados en la hoja de recolección de datos.

Para dar respuesta a los objetivos planteados, los datos obtenidos fueron descritos y analizados según el tipo de variable. Se calculó la media y la desviación estándar de las variables continuas; en el caso de las variables discretas, se calcularon las medianas e identificaron los valores mínimo y máximo. La comparación de las variables continuas entre estadios de Tanner y grupos de edades, se realizó con la prueba de análisis de varianza de una vía (ANOVA). En el caso de las variables discretas, se aplicó la prueba no paramétrica $\mathrm{H}$ de Kruskal-Wallis. La comparación de variables nominales consistió en aplicar la prueba chi-cuadrado de Pearson. Se consideró un valor estadísticamente significativo si $\mathrm{p}<0,05$. Los datos fueron analizados con SPSS 24.

\section{RESULTADOS}

La muestra obtenida fue de 113 pacientes que cumplían con los criterios de inclusión.

La media y desviación estándar (DE) de las medidas uterinas obtenidas en relación con los estadios de Tanner pueden verse en la tabla 1, donde se refleja que la media de la longitud varió desde 33 en pacientes con Tanner I hasta 52 en aquellas con Tanner IV. En cuanto a la media de la medida anteroposterior varió desde 6 ( $\pm 2 \mathrm{DE})$ en pacientes Tanner I, hasta $21( \pm 4 \mathrm{DE})$ en aquellas con Tanner IV, la medida transversal también fue evaluada, encontrándose medias de 12, 16, 24 y 29 para los estadios I, II, III y IV respectivamente. Las medias de la relación cuerpo cuello obtenidas fueron de 0,9 (DE 0,27) en pacientes con estadio I de Tanner, 1,12 (DE 0,39) en pacientes con estadio II, $1,42(\mathrm{DE} 0,40)$ en pacientes con estadio III y 1,30

Tabla 1. Relación de los estadios de Tanner con las medidas ecográficas de útero

\begin{tabular}{|c|c|c|c|c|c|}
\hline \multirow{2}{*}{ Variables } & \multicolumn{4}{|c|}{ Estadio de Tanner } & \multirow{2}{*}{$\mathrm{p}$} \\
\hline & $\mathrm{I}$ & II & III & IV & \\
\hline $\mathrm{N}$ & 22 & 40 & 42 & 9 & - \\
\hline Cuerpo $(\mathrm{mm}) *$ & $15 \pm 3$ & $19 \pm 6$ & $30 \pm 8$ & $29 \pm 8$ & 0,001 \\
\hline Cuello (mm)* & $18 \pm 3$ & $18 \pm 5$ & $21 \pm 5$ & $23 \pm 3$ & 0,001 \\
\hline Relación $\mathrm{C} / \mathrm{C} *$ & $0,90 \pm 0,27$ & $1,12 \pm 0,39$ & $1,42 \pm 0,40$ & $1,30 \pm 0,37$ & 0,001 \\
\hline Longitud $(\mathrm{mm}) *$ & $33 \pm 4$ & $37 \pm 9$ & $51 \pm 11$ & $52 \pm 9$ & 0,001 \\
\hline Anteroposterior $(\mathrm{mm}) *$ & $6 \pm 2$ & $9 \pm 3$ & $16 \pm 6$ & $21 \pm 4$ & 0,001 \\
\hline Transverso $(\mathrm{mm}) *$ & $12 \pm 4$ & $16 \pm 4$ & $24 \pm 7$ & $29 \pm 5$ & 0,001 \\
\hline Volumen $\left(\mathrm{cm}^{3}\right) *$ & $1,13 \pm 0,91$ & $3,37 \pm 1,67$ & $10,80 \pm 6,87$ & $16,42 \pm 7,01$ & 0,001 \\
\hline Línea endometrial $(\mathrm{mm}) * *$ & $0(0-2)$ & $0(0-3)$ & $3(0-9)$ & $3(0-8)$ & 0,002 \\
\hline
\end{tabular}


(DE 0,37 ) en pacientes con estadio IV. La media del volumen uterino obtenido, varió desde $1,13 \mathrm{~cm}^{3}$ en pacientes catalogadas como Tanner I hasta $16,42 \mathrm{~cm}^{3}$ en pacientes con Tanner IV de mama, encontrándose relación significativa entre las mediciones y el estadio de Tanner de mama $(p=0,001)$. Al evaluar la línea endometrial se observó que varió desde no visualizarse hasta un espesor de $8 \mathrm{~mm}$, con medianas de 0 para los estadios I y II de Tanner de mama y de 3 para los estadios III y IV, con una p de 0,002 .

En todas las pacientes en las que se vio línea endometrial, la misma fue homogénea al igual que el miometrio (tabla 2), donde también se plasman los resultados obtenidos en cuanto a la forma uterina. La forma cilíndrica fue la más frecuente, se vio en 15 pacientes con Tanner I, 34 con Tanner II, 13 con Tanner III y 2 con Tanner IV, mientras que la forma de pera predominó en los estadios III (29) y IV (7) de Tanner de mama.

Al comparar estas variables con los grupos etarios (tabla 3), se observa que la relación cuerpo/cuello presentó una media de 1,07 (DE 0,38) para el grupo de 7 - 9 años, de 1,25 (DE 0,38) para el de 10 - 12 años y
Tabla 2. Relación de los estadios de Tanner con la forma, miometrio y línea endometrial

\begin{tabular}{lcccccc}
\hline \multirow{2}{*}{ Variables } & \multicolumn{5}{c}{ Estadio de Tanner } & \multirow{2}{*}{$\mathrm{p}$} \\
\cline { 2 - 5 } & I & II & III & IV & \\
\hline Forma & 7 & 3 & 0 & 0 & \\
Sable & 15 & 34 & 13 & 2 & \\
Cilíndrico & 0 & 3 & 29 & 7 & \\
$\quad$ Piriforme & & & & & $\mathrm{n} / \mathrm{a}$ \\
Miometrio & 22 & 40 & 42 & 9 & \\
$\quad$ Homogéneo & 0 & 0 & 0 & 0 & \\
$\quad$ Heterogéneo & & & & & $\mathrm{n} / \mathrm{a}$ \\
Línea endometrial & & & & & 0,001 \\
$\quad$ Homogénea & 2 & 12 & 34 & 7 & \\
$\quad$ Heterogénea & 0 & 0 & 0 & 0 & \\
\hline
\end{tabular}

de 1,84 (DE 0,35) para el de 13 - 15 años. En cuanto al volumen uterino, se obtuvieron las siguientes medias: $2,47 \mathrm{~cm}^{3}$ (DE 2,22) para las pacientes de 7 a 9 años, $9,12 \mathrm{~cm}^{3}$ (DE 7,02) para aquellas de 10 a 12 años y de $18,8 \mathrm{~cm}^{3}(\mathrm{DE} 7,2)$ para las pacientes con edades

Tabla 3. Relación de edad con las medidas ecográficas de útero

\begin{tabular}{lcccc}
\hline \multirow{2}{*}{ Variables } & \multicolumn{3}{c}{ Grupos de edades (años) } & \multirow{2}{*}{ p } \\
\cline { 2 - 4 } & 7 a 9 & 10 a 12 & 13 a 15 & \\
\hline $\mathrm{N}$ & 50 & 56 & 7 & - \\
Cuerpo * & $18 \pm 6$ & $26 \pm 9$ & $37 \pm 4$ & 0,001 \\
Cuello * & $18 \pm 5$ & $21 \pm 4$ & $21 \pm 5$ & 0,001 \\
Relación C/C * & $1,07 \pm 0,38$ & $1,25 \pm 0,38$ & $1,84 \pm 0,35$ & 0,001 \\
Longitud * & $36 \pm 9$ & $47 \pm 12$ & $58 \pm 4$ & 0,001 \\
Anteroposterior * & $8 \pm 5$ & $14 \pm 6$ & $20 \pm 6$ & 0,001 \\
Transverso * & $15 \pm 5$ & $22 \pm 7$ & $32 \pm 4$ & 0,001 \\
Volumen * & $2,47 \pm 2,22$ & $9,12 \pm 7,02$ & $18,8 \pm 7,2$ & 0,001 \\
Espesor de línea endometrial ** & $0(0-5)$ & $2(0-9)$ & $4(0-8)$ & 0,001 \\
\hline
\end{tabular}

* Media \pm desviación estándar

** Mediana (mínimo - máximo) 
Tabla 4. Relación de edad con la forma, miometrio y línea endometrial

\begin{tabular}{lcccc}
\hline \multirow{2}{*}{ Variables } & \multicolumn{3}{c}{ Grupos de edades (años) } & \multirow{2}{*}{$\mathrm{p}$} \\
\cline { 2 - 4 } & 7 a 9 & 10 a 12 & 13 a 15 & \\
\hline Forma & 9 & 1 & 0 & \\
Sable & 36 & 27 & 1 & \\
Cilíndrico & 5 & 28 & 6 & \\
$\quad$ Piriforme & & & & $\mathrm{n} / \mathrm{a}$ \\
Miometrio & 50 & 56 & 7 & \\
Homogéneo & 0 & 0 & 0 & \\
$\quad$ Heterogéneo & 0 & & & $\mathrm{n} / \mathrm{a}$ \\
Línea endometrial & & 39 & 4 & \\
Homogénea & 12 & 39 & 0 & \\
Heterogénea & 0 & 0 & & \\
\hline
\end{tabular}

comprendidas entre 13 y 15 años. Al evaluar el espesor de la línea endometrial se obtuvieron las siguientes medianas: 0,2 y 4 para los grupos etarios $7-9,10$ - 12 y 13 - 15 años respectivamente, encontrándose relación significativa ( $\mathrm{p}$ de 0,001$)$.
En cuanto a la forma del útero en relación con los grupos etarios (tabla 4), se obtuvo que 6 de las 7 pacientes del grupo etario de 13 a 15 años el mismo era piriforme y la misma forma en 28 de las pacientes entre 10 y 12 años, siendo la forma cilíndrica la que predominó en las pacientes de edades entre 7 y 9 años $(\mathrm{p}=0,001)$.

El evaluar los volúmenes ováricos de estas pacientes a la ecografía, se observó que hubo relación estadísticamente significativa entre los mismos y tanto el estadio de Tanner de mama (tabla 5) como con la edad (tabla 6), obteniéndose las siguientes medias de los volúmenes de ovario derecho $0,93 \mathrm{~cm}^{3}, 1,57 \mathrm{~cm}^{3}$, $2,85 \mathrm{~cm}^{3}$ y $3,22 \mathrm{~cm}^{3}$ y de ovario izquierdo $0,93 \mathrm{~cm}^{3}$, $1,52 \mathrm{~cm}^{3}, 2,89 \mathrm{~cm}^{3}$ y $2,66 \mathrm{~cm}^{3}$, para los estadios I, II, III y IV de Tanner de mama respectivamente.

Con respecto al patrón ecográfico ovárico según estadio de Tanner de mama y grupo etario, como se puede ver en las tablas 7 y 8 . El patrón que predomina es el patrón no homogéneo en el estadio I de Tanner y el patrón microfolicular a partir del Tanner II.

Tabla 5. Relación de los estadios de Tanner con las medidas ecográficas de ovarios

\begin{tabular}{|c|c|c|c|c|c|}
\hline \multirow{2}{*}{ Variables } & \multicolumn{4}{|c|}{ Estadio de Tanner } & \multirow[b]{2}{*}{$\mathrm{p}$} \\
\hline & I & II & III & IV & \\
\hline \multicolumn{6}{|l|}{ Ovario derecho $*$} \\
\hline Longitud & $16 \pm 6$ & $19 \pm 6$ & $24 \pm 6$ & $24 \pm 6$ & 0,001 \\
\hline Anteroposterior & $9 \pm 3$ & $11 \pm 3$ & $12 \pm 3$ & $13 \pm 3$ & 0,001 \\
\hline Transverso & $11 \pm 3$ & $14 \pm 4$ & $18 \pm 3$ & $18 \pm 1$ & 0,001 \\
\hline Volumen & $0,93 \pm 0,65$ & $1,57 \pm 0,89$ & $2,85 \pm 1,27$ & $3,22 \pm 1,22$ & 0,001 \\
\hline \multicolumn{6}{|l|}{ Ovario izquierdo * } \\
\hline Longitud & $17 \pm 5$ & $19 \pm 6$ & $23 \pm 6$ & $22 \pm 5$ & 0,001 \\
\hline Anteroposterior & $8 \pm 2$ & $10 \pm 2$ & $13 \pm 3$ & $13 \pm 3$ & 0,001 \\
\hline Transverso & $11 \pm 3$ & $15 \pm 4$ & $18 \pm 4$ & $18 \pm 2$ & 0,001 \\
\hline Volumen & $0,93 \pm 0,57$ & $1,52 \pm 0,95$ & $2,89 \pm 1,51$ & $2,66 \pm 0,68$ & 0,001 \\
\hline
\end{tabular}

* Media \pm desviación estándar 


\section{P RODRÍGUEZ ET AL}

Tabla 6. Relación de la edad con las medidas ecográficas de ovarios

\begin{tabular}{lcccc}
\hline \multirow{2}{*}{ Variables } & \multicolumn{3}{c}{ Grupos de edades (años) } & \multirow{2}{*}{$\mathrm{p}$} \\
\cline { 2 - 4 } & 7 a 9 & 10 a 12 & 13 a 15 & \\
\hline Ovario derecho & $18 \pm 6$ & $22 \pm 6$ & $25 \pm 6$ & 0,001 \\
$\quad$ Longitud & $10 \pm 3$ & $12 \pm 3$ & $15 \pm 3$ & 0,001 \\
$\quad$ Anteroposterior & $14 \pm 4$ & $17 \pm 4$ & $19 \pm 3$ & 0,001 \\
$\quad$ Transverso & $1,40 \pm 0,94$ & $2,42 \pm 1,29$ & $3,73 \pm 1,29$ & 0,001 \\
$\quad$ Volumen & $18 \pm 5$ & $22 \pm 7$ & $25 \pm 6$ & 0,001 \\
Ovario izquierdo & $9 \pm 3$ & $11 \pm 3$ & $12 \pm 2$ & 0,001 \\
$\quad$ Longitud & $14 \pm 4$ & $17 \pm 4$ & $20 \pm 5$ & 0,001 \\
$\quad$ Anteroposterior & $1,36 \pm 0,87$ & $2,39 \pm 1,38$ & $3,60 \pm 1,89$ & 0,001 \\
$\quad$ Transverso & & &
\end{tabular}

* Media \pm desviación estándar

Tabla 7. Relación de los estadios de Tanner con el patrón ecográfico de ovarios

\begin{tabular}{lcccccc}
\hline \multirow{2}{*}{ Patrón } & \multicolumn{5}{c}{ Estadio de Tanner } & \multirow{2}{*}{$\mathrm{p}$} \\
\cline { 2 - 5 } & I & II & III & IV & \\
\hline Ovario derecho & & & & & 0,005 \\
Homogéneo & 1 & 2 & 0 & 0 & \\
No homogéneo & 11 & 10 & 3 & 0 & \\
Microfolicular & 10 & 28 & 37 & 9 & \\
Folicular & 0 & 0 & 2 & 0 & \\
Ovario izquierdo & & & & & 0,003 \\
Homogéneo & 1 & 1 & 0 & 0 & \\
No homogéneo & 9 & 14 & 3 & 0 & \\
Microfolicular & 12 & 25 & 39 & 9 & \\
Folicular & 0 & 0 & 0 & 0 & \\
\hline
\end{tabular}

Tabla 8. Relación de la edad con patrón ecográfico de ovarios

\begin{tabular}{lcccc}
\hline \multirow{2}{*}{ Patrón } & \multicolumn{3}{c}{ Grupos de edades (años) } & \multirow{2}{*}{$\mathrm{p}$} \\
\cline { 2 - 4 } Ovario derecho & & 10 a 12 & 13 a 15 & \\
\hline Homogéneo & 3 & 0 & 0 & \\
No homogéneo & 17 & 7 & 0 & \\
Microfolicular & 0 & 47 & 7 & \\
Folicular & 0 & 2 & 0 & \\
Ovario izquierdo & & & & 0,004 \\
Homogéneo & 2 & 0 & 0 & \\
No homogéneo & 19 & 7 & 0 & \\
Microfolicular & 29 & 49 & 7 & \\
Folicular & 0 & 0 & 0 & \\
\hline
\end{tabular}




\section{DISCUSIÓN}

Resulta de mucha importancia estandarizar las medidas ecográficas de los órganos pélvicos en niñas saludables al momento de identificar alguna alteración en el normal desarrollo en esta población, a pesar de que se han realizado estudios evaluando dichas medidas, actualmente no se ha llegado a un consenso con respecto a estos valores.

En este estudio se encontró que existe una relación estadísticamente significativa entre las medidas uterinas y los volúmenes ováricos con el estadio de Tanner de mama y la edad y que dichas medidas progresaron a medida que avanzaban los parámetros con los que fueron comparados, al igual que lo encontrado por otros autores $(11,15$ - 18). El mayor crecimiento uterino fue observado en la transición de estadio III y IV de Tanner, lo que coincide con los hallazgos de Seth y cols. (15) pero difiere con Razzaghy-Azar y cols. (13) quienes encontraron un crecimiento continuo desde estadio I al IV y este crecimiento fue más acentuado en el estadio IV.

Similar evolución se observa al comparar los volúmenes ováricos con el estadio de Tanner, sin embargo, en este punto, Seth y cols. (15) consiguieron que a un crecimiento inicial le siguieron valores estables mientras que Griffin y cols. (19) encontraron que el volumen ovárico aumentaba exponencialmente con la edad, pero no observaron relación con el estadio puberal.

En estudios como el de Herter y cols. (12) y Bridges y cols. (20), la forma uterina fue evaluada para determinar su valor como marcador puberal, encontrando que dicho parámetro y la relación cuerpo/cuello son mejores marcadores que las medidas uterinas. En este estudio, la forma de pera típica del adulto predominó en los estadios III y IV de Tanner de mama y la relación cuerpo/cuello fue mayor en dichos estadios. Razzaghy-
Azar y cols. (13) describieron la visualización del endometrio como una línea ecogénica después de los 7 años de edad, en la presente serie, se vio este tipo de línea endometrial en solo 2 pacientes con estadio I de Tanner (mediana de 0 y valor máximo de $2 \mathrm{~mm}$ ) y en 12 pacientes en el grupo etario de 7 a 9 años (mediana de 0 y valor máximo de $5 \mathrm{~mm}$ ).

Se ha descrito que el patrón ovárico es indicativo de la función folicular gonadotropina dependiente, por lo que se establece que la presencia de folículos ováricos está relacionada con la estimulación folicular por un aumento en los niveles hormonales, encontrándose en el presente estudio que el patrón que predominó fue el microfolicular, con diferencias menores en pacientes con estadio I de Tanner, lo que difiere de Herter y cols. (12) quienes observaron que el patrón homogéneo predominó en pacientes sin telarquia, pudiendo explicarse porque ellos incluyeron niñas en edades prepuberales las cuales no eran objetivo de estudio en este caso.

En conclusión, en el presente estudio se demostró que tanto el útero como los ovarios presentan crecimiento continuo en relación a la edad y al estadio de Tanner de mama, siendo de importancia comparar ambos parámetros al momento de evaluar las medidas ecográficas de dichos órganos en niñas.

\section{REFERENCIAS}

1. Rodríguez MJ, Hernández I, Parera N. Patología ginecológica de la infancia y la adolescencia. En: Kauslaskas S, Zapardiel I, editores. Fundamentos de Ginecología. 3ra ed. Barcelona: Panamericana; 2009. p. 265-274.

2. Ward V. Técnicas de imagen. En: Emans S, Laufer M, editores. Ginecología en la Infancia y la Adolescencia. 6ta ed. Barcelona: Lippincott; 2013. p. 421-446

3. Possiel M. Fisiología del desarrollo puberal en niñas $\mathrm{y}$ evaluación hormonal en ginecología pediátrica y adolescente. En: Lewitan G, editora. Ginecología de 
Niñas y Adolescentes: Diagnóstico, Tratamiento y Prevención. 1a ed. Buenos Aires: Journal; 2013. p. 3542.

4. Barrio R, Roldán B, Martín-Frías M. Inducción de la pubertad. Rev Esp Endocrinol Pediatr. 2015; 6(Supp1): 39-44.

5. Speroff L, Fritz M. Anomalías de la pubertad y problemas del crecimiento. En: Speroff L, Fritz M, editores. Endocrinología clínica y esterilidad. $7 \mathrm{ma}$ ed. Philadelphia: Lippincott Williams y Wilkins; 2006. p. 362-400.

6. Pitts S, Gordon K. Fisiología de la pubertad. En: Emans S, Laufer M, editores. Ginecología en la Infancia y la Adolescencia. 6ta ed. Barcelona: Lippincott; 2013. p. 100-113.

7. Sileo E. Pubertad: variantes normales, patológicas y características principales. En: Terán J, Febres F, editores. Endocrinología ginecológica y reproducción humana. Caracas: Ateproca; 1995. p. 175-205.

8. Poncelas M. Conceptos generales sobre ecografía ginecológica en pediatría. En: Lewitan G, editora. Ginecología de Niñas y Adolescentes: Diagnóstico, Tratamiento y Prevención. 1a ed. Buenos Aires: Journal; 2013. p. 25-34.

9. Unshelm P, Iturriza T. Ultrasonido de la pelvis pediátrica. En: Sánchez E, editora. Ginecología Infantojuvenil. $1^{\mathrm{a}}$ ed. Buenos Aires: Médica Panamericana; 2011. p. 3749.

10. Sánchez B. Pubertad precoz. En: Sánchez E, editora. Ginecología Infantojuvenil. $1^{\text {a }}$ ed. Buenos Aires: Médica Panamericana; 2011. p. 334-347.

11. Salardi S, Orsini L, Cacciari E, Bovicelli L, Tassoni P, Reggiani A. Pelvic ultrasonography in premenarcheal girls: relation to puberty and sex hormone concentrations. Arch Dis Child. 1985; 60(2): 120-125.

12. Herter L, Golendziner E, Monteiro J, Becker E, Spritzer P. Ovarian and uterine sonography in healthy girls between 1 and 13 years old: correlation of findings with age and pubertal status. Am J Roentgenol. 2002; 178(6):1531-1536.
13. Razzaghy-Azar M, Ghasemi F, Hallaji F, Ghasemi A, Ghasemi M. Sonographic measurement of uterus and ovaries in premenarcheal healthy girls between 6 and 13 years old: correlation with age and pubertal status. J Clin Ultrasound 2011; 39(2):64-73.

14. Caraballo A, Sánchez B, Perera A, M Pérez, De Abreu L, Gónzález L, et al. Ultrasonido pélvico transabdominal en prepúberes y adolescentes. Utilidad práctica. Arch Venez Pueric Pediatr. 2002; 65(3): 100-105.

15. Seth A, Aggarwal A, Sandesh K, Solanki RS, Aneja S, Kumar G. Pelvic ultrasonography in pubertal girls. Indian J Pediatr. 2002; 69(10):869-872.

16. Ivarsson A, Nilsson K, Persson P. Ultrasonography of the pelvic organs in prepuderal and postpuberal girls. Arch Dis Child. 1983; 58(5):352-354.

17. Buzi F, Pilotta A, Dordoni D, Lombardi A, Zaglio $\mathrm{S}$, Adlard P. Pelvic ultrasonography in normal girls and with pubertal precocity. Acta Paediatr. 1998; 87(11):1138-1145.

18. Orbak Z, Sağsöz N, Alp H, Tan H, Yildirim H, Kaya D. Pelvic ultrasound measurements in normal girls: relation to puberty and sex hormone concentation. J Pediatr Endocrinol Metab. 1998; 11(4):525-530.

19. Griffin IJ, Cole TJ, Duncan KA, Hollman AS, Donaldson MD. Pelvic ultrasound measurements in normal girls. Acta Paediatr. 1995; 84(5):536-543.

20. Bridges NA, Cooke A, Healy MJ, Hindmarsh PC, Brook CG. Growth of the uterus. Arch Dis Child. 1996; 75(4):330-331. 